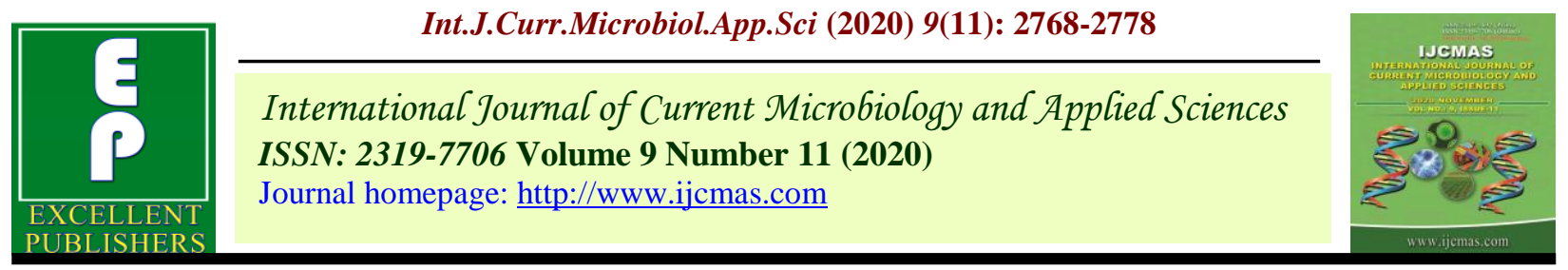

Original Research Article

https://doi.org/10.20546/ijcmas.2020.911.336

\title{
Identification of Different Land Use Systems in Leh Region of Himalayan Cold Desert
}

\author{
Jigmet Namgial ${ }^{1}$, Mukesh Prabhakar ${ }^{1}$, RohitBishist ${ }^{1}$, Krishan Lal Gautam ${ }^{1 *}$, \\ Harish Sharma ${ }^{1}$ and Archana Sharma ${ }^{2}$ \\ ${ }^{1}$ Department of Silviculture and Agroforestry Dr Y S Parmar University of Horticulture and \\ Forestry NauniSolan Himachal Pradesh, India \\ ${ }^{2}$ Department of Soil Science and Water Management Dr Y S Parmar University of \\ Horticulture and Forestry Nauni, Solan Himachal Pradesh, India \\ *Corresponding author
}

\section{A B S T R A C T}

\section{Keywords}

Agroforestry,

Farmers,

Himalayan,

Household, Land

use system

Article Info

Accepted:

20 October 2020

Available Online:

10 November 2020

\begin{abstract}
Study was carried out to identify the different land use systems in Leh region of Himalayan cold desert was conducted in two blocks of Leh district of Jammu and Kashmir. The study sites were selected through multi stage random sampling technique. Khaltsi and Saspol block were selected for the study and from each block 36 households were selected and a total of 72 households were selected from both the blocks. The primary data about the study was collected from the household itself. The systems identified are agriculture, horticulture, agrisilviculture, agrihorticulture, silvipasture, hortisilvipasture and agrihorticulture. A total number of 21 and 19 species of trees, grasses and agricultural crops were recorded from the different farmer's field of Khaltsi and Saspol block respectively. Thus, concluded that that farmers in Leh region practiced different land use systems viz. agriculture, horticulture, agrisilviculture, agrihortisilviculture, hortisilvipasture, silvipasture and agrihorticulture.
\end{abstract}

\section{Introduction}

Land is the most important natural resource which embodies soil, water and associated flora and fauna involving total ecosystem. The term 'land use' refers to the human activity or economic function associated with a specific piece of land whereas the "land cover' relates to the type of feature present on the surface of the earth. Information on land use or land cover allows a better understanding of the land utilization aspects like cropping patterns, fallow lands, forests, pasture lands, wastelands and surface waterbodies which are vital for development planning (Lillesand and Kiefer, 2000). North western Himalaya is basically an agroecosystem, where 90 per cent of its total population lives in villages whose economy is dependent on agriculture, horticulture and animal husbandry (Atul and Khosla, 1994). The species composition varies depending on land holdings and basic requirement of the farmers (Toky et al., 1989). In India, cold 
desert comes under the trans Himalayan zone which is approximately 1,03,11,300 hectares of area (Gupta and Arora, 2016). In India, major part of cold desret in the country is confirmed to Ladakh with Approximately 82,665 square $\mathrm{Km}$ area. The Ladakh region consists of 2 districts, namely Leh and Kargil. Land based economy of this region consists of agriculture and allied sectors.

The Himalaya is ecologically fragile and subsistence agriculture is the backbone of local livelihoods. Agroforestry in this region has a potential to provide options for improvement in livelihood through simultaneous production of food, fodder and firewood. Agroforestry is one of the important terrestrial carbon sequestration systems that help in mitigation of the impact of climate change (Tiwari, 2000).

\section{Materials and Methods}

Present study was carried out in two blocks i.e. Khaltsi and Saspol block of Leh district of Jammu and Kashmir. This region is enclosed by the Ladakh and Karakoram range in the north and Zanskar mountains and The Great Himalaya in the south (Fig. 1). The average altitude of the region is $3200 \mathrm{~m}$ amsl. Precipitation is very low and mainly occurs in the form of snow in the winter months.

The study sites were selected through multi stage random sampling technique. Leh district consisted of 16 blocks and out of all the blocks, Khaltsi and Saspol block were selected for the study. From each block 36 households were selected and a total of 72 households were selected from both the blocks for the study. The primary data about the study was collected from the household itself.

The selected farmers were first interviewed regarding the details of the different crops being cultivated by them and were noted down. Fields of the farmers were visited and then on the principal and associated components, the different land use systems were identified. Agroforestry systems existing in the study area were further classified on the basis of structure (nature and arrangement) and function (role/output) of components as suggested by Nair (1985). Primary and secondary components of each system type were identified after recognizing the structure of the system and specific function of the components (Table 1 and 2).

\section{Primary components}

The component which occupied the larger area of the total unit area and serve the major function i.e. production of primary outputs needed by the farmers.

\section{Secondary component}

The component, which occupied relatively lesser area of the total unit area compared to the area under primary component and yielding secondary outputs needed by the farmers.

\section{Results and Discussion}

In the study area, a total of seven land use systems viz., agriculture, horticulture, agrisilviculture, agrihortisilviculture, silvipasture, hortisilvipasture and agrihorticulture were identified in the fields of different farmer's category. The climatic conditions of the area allow a very short growing period which extends from April to September. Therefore, the farmers of the region harvest single crop a year i.e. Kharif season crops. These systems may be attributed to agro climatic conditions of the area and needs of the farmer i.e. food, fodder, fuel wood and timber. 
In Khaltsi block, on the fields of marginal, small and medium categories of farmers, total system units in agriculture land use were 8,10 and 20; in agrisilviculture land use, total system units were 6,7 and 11 . The system units in agrihortisilviculture land use were 12, 15 and 21 similarly in case of hortisilvipasture land use, total system units were 5, 10 and 14 . However horticulture, silvipasture and agrihorticulture land use system was present only in case of small and medium category of farmers with respective system units (3 and 6), (11 and15) and (9 and 10) (Table 3).

Results reveal in Table 4 that in agriculture land use system, cereal crops grown wereWheat, Foxtail Millet, Buckwheat and Barley; vegetable crops consisted of Cauliflower, Cabbage, Pea, Turnip, Beans, Onion, Tomato and Potato. In this land use system, commonly a single field was cropped in parts by planting various crop combinations - (i) Buckwheat + Foxtail Millet (ii) Pea + Potato + Beans (iii) Wheat + Turnip+ Onion (iv) Cabbage + Cauliflower + Beans + Tomato Some of the agricultural fields were planted with sole crops of Wheat and Barley. In horticulture land use system, the constituent fruit tree species were Apricot, Apple, Mulberry and Walnut. In this land use system the main fruit tree species was Apricot that was either managed as pure orchard or other fruit tree species- Apple, Mulberry and Walnut were also grown along with it by the farmers. In agrisilviculture land use system, agriculture crops were cereals- Wheat, Buckwheat, Foxtail Millet and Barley; oil seeds- mustard; vegetables- Pea, Turnip, and Potato. It was recorded that in all the system units trees of Populus nigra and Salix albawere retained by farmers on the bunds of their field.

In agrihortisilviculture land use system, the farmers of Khaltsi block farmed six main crops i.e. Wheat, Cabbage, Cauliflower,
Turnip, Rajmash and Pea. The tree species of Populus nigra and Salix alba were retained by farmers on the bunds of their field whereas fruit tree species of Apricot and Apple were planted by the farmers across their agricultural fields. The tree components in silvipasture land use system were: Populus nigra and Salix alba. The pasture components consisted of Medica gosativa, Medica gofalcata and Iris lactea. The different tree species in hortisilvipasture land use system were Prunus armeniaca, Malus domestica, Populus nigra and Salix alba, whereas, Medica gosativa, Medica gofalcata and Iris lacteal constituted the pasture component. In agrihorticulture land use system, the farmers mainly raised six agriculture crops viz. Wheat, Potato, Pea, Beans, Turnip and Tomato. The tree component comprised of Apricot and Apple (Table 4).

\section{Saspol block}

In Saspol block, on the fields of marginal, small and medium categories of farmers, total system units in agriculture land use were 5, 7 and 14; in agrisilviculture land use, total system units were 5, 6 and 9. The system units in agrihortisilviculture land use were 8 , 9 and 24 and in case of hortisilvipasture land use, total system units were 4, 15 and 11 . The horticulture and agrihorticulture land use systems were present only in case of medium category of farmers with system units of 4 and 8 whereas, silvipasture land use system was present only in small and medium category of farmer with system units of 8 and 10. (Table 3)

Result reveal (Table 5) thatin agriculture land use system, cereals crops grown were- Wheat, and Barley; oil seed and pulses- Mustard and Rajmash; vegetable crops consisted of Cauliflower, Cabbage, Pea, Turnip, Beans, Onion, Tomato and Potato. In this land use system commonly a single field was cropped 
in parts by planting various crop combinations (i) Pea + Potato + Tomato + Beans (ii) Wheat + Turnip+ Cabbage (iii) Mustard + Cauliflower + Beans + Rajmash. Some of the agricultural fields were planted with sole crops of Wheat and Barley. In horticulture land use system, the constituent fruit tree species were Apricot, Apple, Mulberry and Walnut. In this land use system the main fruit tree species was Apricot that was either managed as pure orchard or other fruit tree species - Apple and Walnut were also grown along with it by the farmers. In agrisilviculture land use system, agriculture crops were cereals- Wheat and Barley; oil seed- Mustard; vegetables- Pea, Turnip,
Beans, and Potato. It was recorded that in all the system units trees of Populus nigra and Salix albawere retained by farmers on the bunds of their field.

In agrihortisilviculture land use system, the farmers of Saspol block mainly farmed six main crops i.e. Wheat, Barley, Turnip, Potato, Beans and Pea. The tree species of Populus nigra and Salix alba were retained by farmers on the bunds of their field whereas tree species of Apricot, Apple and Mulberry were planted by the farmers across their agricultural fields. The tree components in this system were: Populus nigra and Salix alba.

Table.1 Locality factors of the study area

\begin{tabular}{|c|c|}
\hline Latitude & $\mathbf{3 4}^{\circ} \mathbf{1 0} \boldsymbol{\aleph}^{\mathbf{N}}$ \\
\hline Longitude & $\mathbf{7 7}^{\circ} \mathbf{3 5} \boldsymbol{\text { E }}$ \\
\hline Altitude & $\mathbf{2 9 0 0 - 3 5 0 0} \mathbf{~ m}$ \\
\hline Climate type & Dry temperate \\
\hline Soil Texture & Coarse and sandy \\
\hline
\end{tabular}

Table.2 Multi-stage random sampling method for choosing the study sites

\begin{tabular}{|c|c|c|c|c|}
\hline $\begin{array}{l}\text { Sr. } \\
\text { no. }\end{array}$ & Block & Panchayat & Village & $\begin{array}{l}\text { Farmer ( } 9 \text { farmers from each village } \\
\text { according to their land holding ) }\end{array}$ \\
\hline \multirow[t]{2}{*}{1.} & \multirow[t]{2}{*}{ Khaltsi } & Khaltsi & $\begin{array}{cl}\text { I. } & \text { Khaltsi } \\
\text { II. } & \text { Skindiyang }\end{array}$ & $\begin{array}{l}\text { Three marginal farmer }(<1 \text { hectare }) \\
\text { Three small farmer }(1-2 \text { hectare }) \\
\text { Three medium farmer }(2-5 \text { hectare })\end{array}$ \\
\hline & & Tingmosgang & $\begin{array}{ll}\text { I. } & \text { Tingmosgang } \\
\text { II. Nurla }\end{array}$ & $\begin{array}{l}\text { Three marginal farmer }(<1 \text { hectare }) \\
\text { Three small farmer }(1-2 \text { hectare }) \\
\text { Three medium farmer }(2-5 \text { hectare })\end{array}$ \\
\hline \multirow[t]{2}{*}{2.} & \multirow[t]{2}{*}{ Saspol } & Saspol & $\begin{array}{cl}\text { I. } & \text { Saspol } \\
\text { II. } & \text { Saspochey }\end{array}$ & $\begin{array}{l}\text { Three marginal farmer }(<1 \text { hectare }) \\
\text { Three small farmer }(1-2 \text { hectare }) \\
\text { Three medium farmer }(2-5 \text { hectare })\end{array}$ \\
\hline & & Gera & $\begin{array}{cc}\text { I. } & \text { Gera } \\
\text { II. } & \text { Alchi }\end{array}$ & $\begin{array}{l}\text { Three marginal farmer }(<1 \text { hectare }) \\
\text { Three small farmer }(1-2 \text { hectare }) \\
\text { Three medium farmer }(2-5 \text { hectare })\end{array}$ \\
\hline
\end{tabular}


Table.3 System units in different land use systems in Khaltsi block and Saspol block under different farmers' category

\begin{tabular}{|c|c|c|c|c|c|c|}
\hline \multirow[t]{3}{*}{$\begin{array}{l}\text { Land use } \\
\text { Systems }\end{array}$} & \multicolumn{3}{|c|}{$\begin{array}{c}\text { Farmers category in } \\
\text { Khaltsi block }\end{array}$} & \multicolumn{3}{|c|}{$\begin{array}{c}\text { Farmers category in } \\
\text { Saspol block }\end{array}$} \\
\hline & Marginal & Small & Medium & $\underset{1}{\text { Margina }}$ & Small & $\underset{\mathbf{m}}{\text { Mediu }}$ \\
\hline & \multicolumn{6}{|c|}{ Number of system units } \\
\hline Agriculture & 8 & 10 & 20 & 5 & 7 & 14 \\
\hline Horticulture & - & 3 & 6 & - & - & 4 \\
\hline Agrisilviculture & 6 & 7 & 11 & 5 & 6 & 9 \\
\hline Agrihortisilviculture & 12 & 15 & 21 & 8 & 9 & 24 \\
\hline Silvipasture & - & 11 & 15 & - & 8 & 10 \\
\hline Hortisilvipasture & 5 & 10 & 14 & 4 & 15 & 11 \\
\hline Agrihorticulture & - & 9 & 10 & - & - & 8 \\
\hline
\end{tabular}

Table.4 System units and their constituents under different land use systems practiced by farmers of Khaltsi block

\begin{tabular}{|c|c|c|}
\hline \multicolumn{3}{|c|}{ Agriculture land use system } \\
\hline Sr. no. & \multicolumn{2}{|c|}{ Agriculture crops } \\
\hline 1 & \multicolumn{2}{|l|}{ Triticum aestivum(Wheat) } \\
\hline 2 & \multicolumn{2}{|l|}{ Hordeum vulgare(Barley) } \\
\hline 3 & \multicolumn{2}{|c|}{ Fagopyrum esculentum(Buckwheat) + Setaria italica(Foxtail Millet) } \\
\hline 4 & \multicolumn{2}{|c|}{ Pisum sativum(Pea) +Solanum tuberosum(Potato) + Phaseolus vulgaris (Beans) } \\
\hline 5 & \multicolumn{2}{|c|}{ Triticum aestivum(Wheat) + Brassica rapa(Turnip) + Allium sepa(Onion) } \\
\hline 6 & \multicolumn{2}{|c|}{$\begin{array}{l}\text { Brassica oleracea var. botrytis (Cabbage) + Brassica oleracea var. capitata (Cauliflower) } \\
+ \text { Phaseolus vulgaris (Beans) + Solanum lycopersicon (Tomato) }\end{array}$} \\
\hline \multicolumn{3}{|c|}{ Horticulture Land use system } \\
\hline Sr. No. & \multicolumn{2}{|c|}{ Fruit trees } \\
\hline 1 & \multicolumn{2}{|l|}{ Prunus armeniaca(Apricot) } \\
\hline 2 & \multicolumn{2}{|c|}{ Prunus armeniaca(Apricot) + Malus domestica(Apple) + Morus alba (Mulberry) } \\
\hline 3 & \multicolumn{2}{|c|}{ Prunus armeniaca(Apricot) + Malus domestica(Apple) + Juglansregia(Walnut) } \\
\hline \multicolumn{3}{|c|}{ Agrisilviculture land use system } \\
\hline Sr. No. & \multirow{2}{*}{$\begin{array}{l}\text { Agriculture crop } \\
\text { Triticum aestivum (Wheat) }\end{array}$} & Fodder/Timber/Fuelwood \\
\hline 1 & & $\begin{array}{l}\text { Populus nigra(Poplar) }+ \text { Salix alba } \\
\text { (Willow) }\end{array}$ \\
\hline 2 & Triticum vulgare (Barley) & $\begin{array}{l}\begin{array}{l}\text { Populus nigra(Poplar) }+ \text { Salix alba } \\
\text { (Willow) }\end{array}\end{array}$ \\
\hline 3 & $\begin{array}{l}\text { Fagopyrum esculentum(Buckwheat) }+ \\
\text { Setaria millet (Foxtail Millet) }\end{array}$ & $\begin{array}{l}\text { Populus nigra(Poplar) }+ \text { Salix alba } \\
\text { (Willow) }\end{array}$ \\
\hline 4 & $\begin{array}{l}\text { Pisum sativum }(\mathrm{Pea})+\text { Solanum tuberosum } \\
\text { (Potato) }\end{array}$ & $\begin{array}{l}\begin{array}{l}\text { Populus nigra(Poplar) } \\
\text { (Willow) }\end{array} \\
\text { (Walix alba }\end{array}$ \\
\hline 5 & $\begin{array}{l}\text { Brassica rapa (Turnip) + Brassica juncea } \\
\text { (Mustard) }\end{array}$ & $\begin{array}{l}\text { Populus nigra (Poplar) }+ \text { Salix alba } \\
\text { (Willow) }\end{array}$ \\
\hline
\end{tabular}




\begin{tabular}{|c|c|c|}
\hline \multicolumn{3}{|c|}{ Agrihortisilviculture land use system } \\
\hline \multirow{2}{*}{$\begin{array}{c}\text { Sr. no. } \\
1\end{array}$} & \multirow{2}{*}{$\begin{array}{l}\text { Agriculture crop } \\
\text { Triticum aestivum (Wheat) }\end{array}$} & \multirow{2}{*}{ 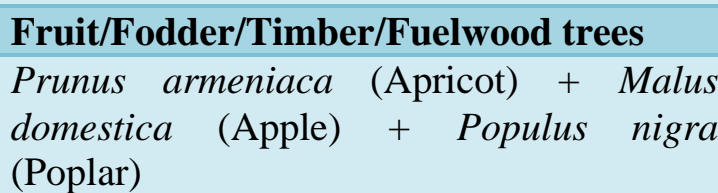 } \\
\hline & & \\
\hline 2 & $\begin{array}{l}\text { Pisum sativum }(\mathrm{Pea})+\text { Brassica } \\
\text { rapa(Turnip) }\end{array}$ & $\begin{array}{l}\text { Prunus armeniaca }(\text { Apricot })+\text { Populus } \\
\text { nigra }(\text { Poplar })+\text { Salix alba }(\text { Willow })\end{array}$ \\
\hline 3 & $\begin{array}{l}\text { Brassica oleraceavar. botrytis (Cabbage) + } \\
\text { Brassica oleracea var. Capitata } \\
\text { (Cauliflower) + Pisum sativum (Pea) }\end{array}$ & \multirow{2}{*}{$\begin{array}{l}\text { Prunus armeniaca(Apricot)+ Populus } \\
\text { nigra(Poplar) } \\
\begin{array}{l}\text { Prunus armeniaca(Apricot) }+ \text { Populus } \\
\text { nigra(Poplar) }+ \text { Salix alba (Willow) }\end{array}\end{array}$} \\
\hline 4 & $\begin{array}{l}\text { Brassica rapa (Turnip) }+ \text { Phaseolus } \\
\text { vulgaris (Rajmash) }\end{array}$ & \\
\hline \multicolumn{3}{|c|}{ Silvipasture land use system } \\
\hline Sr. No. & Fodder/Timber/Fuelwood trees & \multirow{2}{*}{$\begin{array}{l}\text { Grasses } \\
\text { Medicago sativa (Alfalfa) }+ \text { Medicago } \\
\text { falcate (Alfalfa) + Iris lacteal (Iris) }\end{array}$} \\
\hline 1 & Populus nigra (Poplar) & \\
\hline 2 & $\begin{array}{l}\text { Populus nigra(Poplar) }+ \text { Salix alba } \\
\text { (Willow) }\end{array}$ & $\begin{array}{l}\text { Medicago sativa (Alfalfa) }+ \text { Medicago } \\
\text { falcate (Alfalfa) }\end{array}$ \\
\hline 3 & $\begin{array}{l}\text { Populus nigra(Poplar) } \\
\text { (Willow) }\end{array}$ & Medicago sativa (Alfalfa) \\
\hline \multicolumn{3}{|c|}{ Hortisilvipasture land use system } \\
\hline Sr. No. & Fruit/Fodder/Timber/Fuelwood trees & Grasses \\
\hline 1 & $\begin{array}{l}\text { Malus domestica (Apple) }+ \text { Populus } \\
\text { nigra(Poplar) }\end{array}$ & $\begin{array}{l}\text { Medicago sativa (Alfalfa) }+ \text { Medicago } \\
\text { falcata(Alfalfa) }\end{array}$ \\
\hline 2 & $\begin{array}{l}\text { Prunus armeniaca (Apricot) + Malus } \\
\text { domestica }(\text { Apple })+\text { Populus nigra(Poplar) } \\
+ \text { Salix alba (Willow) }\end{array}$ & Medicago sativa (Alfalfa) + Iris lactea(Iris) \\
\hline 3 & $\begin{array}{l}\text { Prunus armeniaca(Apricot) }+ \text { Juglans } \\
\text { regia }(\text { Walnut })+\text { Salix alba (Willow) }\end{array}$ & Medicago sativa (Alfalfa) \\
\hline 4 & $\begin{array}{l}\text { Prunus armeniaca(Apricot) + Salix alba } \\
\text { (Willow) + Morus alba (Mulberry) }\end{array}$ & $\begin{array}{l}\text { Medicago falcata(Alfalfa) } \\
\text { lactea(Iris) }\end{array}$ \\
\hline \multicolumn{3}{|c|}{ Agrihorticulture land use system } \\
\hline Sr. No. & \multirow{2}{*}{$\begin{array}{l}\text { Agriculture crop } \\
\text { Triticum aestivum (Wheat) }\end{array}$} & Fruit trees \\
\hline 1 & & $\begin{array}{l}\text { Prunus armeniaca(Apricot) + Malus } \\
\text { domestica (Apple) }\end{array}$ \\
\hline 2 & $\begin{array}{l}\text { Solanum tuberosum (Potato) + Pisum } \\
\text { sativum (Pea) }+ \text { Phaseolus vulgaris (Beans) }\end{array}$ & \multirow{2}{*}{$\begin{array}{l}\text { Prunus armeniaca (Apricot) + Malus } \\
\text { domestica(Apple) } \\
\text { Prunus armeniaca(Apricot) }\end{array}$} \\
\hline 3 & $\begin{array}{l}\text { Brassica rapa(Turnip) } \\
\text { lycopersicon(Tomato) }\end{array}+$ Solanum & \\
\hline
\end{tabular}


Table.5 System units and their constituents under different land use systems practiced by farmers of Saspol block

\begin{tabular}{|c|c|c|}
\hline \multicolumn{3}{|c|}{ Agriculture land use system } \\
\hline Sr. No. & \multicolumn{2}{|c|}{ Agriculture crops } \\
\hline 1 & \multicolumn{2}{|l|}{ Triticum aestivum(Wheat) } \\
\hline 2 & \multicolumn{2}{|c|}{ Hordeum vulgare (Barley) } \\
\hline 3 & \multicolumn{2}{|c|}{$\begin{array}{l}\text { Solanum tuberosum(Potato })+ \text { Pisum sativum }(\text { Pea })+\text { Phaseolus vulgaris }(\text { Beans })+\text { Solanum } \\
\text { lycopersicon }(\text { Tomato })+\text { Allium sepa }(\text { Onion })\end{array}$} \\
\hline 4 & \multicolumn{2}{|c|}{$\begin{array}{l}\text { Triticum aestivum(Wheat) + Brassica rapa(Turnip) + Brassica oleraceavar. botrytis } \\
\text { (Cabbage) }\end{array}$} \\
\hline 5 & \multicolumn{2}{|c|}{$\begin{array}{l}\text { Phaseolus vulgaris (Beans) + Brassica oleraceavar. capitata(Cauliflower) + Brassica } \\
\text { juncea(Mustard) + Phaseolus vulgaris (Rajmash) }\end{array}$} \\
\hline \multicolumn{3}{|c|}{ Horticulture Land use system } \\
\hline Sr. No. & \multicolumn{2}{|c|}{ Fruit trees } \\
\hline 1 & \multicolumn{2}{|c|}{ Prunus armeniaca(Apricot) } \\
\hline 2 & \multicolumn{2}{|c|}{ Prunus armeniaca(Apricot) + Malus domestica(Apple) } \\
\hline 3 & \multicolumn{2}{|c|}{ Prunus armeniaca(Apricot) + Malus domestica(Apple) + Juglansregia(Walnut) } \\
\hline \multicolumn{3}{|c|}{ Agrisilviculture land use system } \\
\hline Sr. No. & Agriculture crop & Fodder/Timber/Fuelwood \\
\hline 1 & Triticum aestivum(Wheat) & Populus nigra(Poplar) + Salix alba (Willow) \\
\hline 2 & $\begin{array}{l}\text { Brassica rapa(Turnip) }+ \text { Brassica } \\
\text { juncea(Mustard) }\end{array}$ & Populus nigra(Poplar) + Salix alba (Willow) \\
\hline 3 & $\begin{array}{l}\text { Triticum aestivum(Wheat) }+ \text { Brassica } \\
\text { rapa(Turnip) }\end{array}$ & Populus nigra(Poplar) \\
\hline 4 & 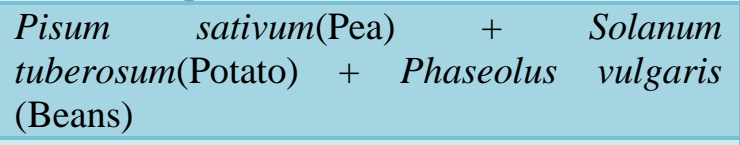 & Populus nigra (Poplar) + Salix alba (Willow) \\
\hline 5 & Triticum vulgare(Barley) & Populus nigra(Poplar) + Salix alba (Willow) \\
\hline \multicolumn{3}{|c|}{ Agrihortisilviculture land use system } \\
\hline 1 & Triticum aestivum(Wheat) & $\begin{array}{l}\text { Malus domestica(Apple) }+\underset{\text { Prunus }}{\text { armeniaca(Apricot) }+ \text { Populus nigra(Poplar) }}\end{array}$ \\
\hline 2 & Triticum vulgare (Barley) & $\begin{array}{l}\text { Prunus armeniaca(Apricot) }+ \text { Populus } \\
\text { nigra(Poplar) }+ \text { Salix alba (Willow) }\end{array}$ \\
\hline 3 & $\begin{array}{l}\text { Solanum tuberosum (Potato) }+ \text { Pisum } \\
\text { sativum }(\text { Pea })\end{array}$ & $\begin{array}{l}\text { Prunus armeniaca(Apricot) + Morus alba } \\
\text { (Mulberry) }+ \text { Populus nigra(Poplar) }\end{array}$ \\
\hline 4 & $\begin{array}{l}\text { Brassica rapa(Turnip) + Phaseolus vulgaris } \\
\text { (Beans) }\end{array}$ & $\begin{array}{l}\text { Prunus armeniaca (Apricot) }+ \text { Populus } \\
\text { nigra(Poplar) }+ \text { Salix alba (Willow) }\end{array}$ \\
\hline \multicolumn{3}{|c|}{ Silvipasture land use system } \\
\hline Sr. No. & \multirow{2}{*}{$\begin{array}{l}\text { Fodder/Timber/ } \\
\text { Populus nigra(Poplar) }\end{array}$} & Grasses \\
\hline 1 & & $\begin{array}{l}\text { Medicago sativa (Alfalfa) }+ \text { Medicago } \\
\text { falcata(Alfalfa) }+ \text { Iris lactea }(\text { Iris })\end{array}$ \\
\hline 2 & Populus nigra(Poplar) + Salix alba (Willow) & $\begin{array}{l}\text { Medicago sativa (Alfalfa) }+ \text { Medicago } \\
\text { falcata(Alfalfa) }\end{array}$ \\
\hline 3 & Populus nigra(Poplar) + Salix alba (Willow) & Medicago sativa (Alfalfa) \\
\hline Hortisilvi & asture land use system & \\
\hline Sr. No. & Fodder/Timber/Fuelwood trees & Grasses \\
\hline 1 & Malus domestica(Apple) + Populus nigra & Medicago sativa (Alfalfa) + Medicago \\
\hline
\end{tabular}




\begin{tabular}{|c|c|c|}
\hline & (Poplar) & falcata(Alfalfa) \\
\hline 2 & $\begin{array}{l}\text { Prunus armeniaca (Apricot) }+ \text { Malus } \\
\text { domestica(Apple) }+ \text { Populus nigra(Poplar) }+ \\
\text { Salix alba (Willow) }\end{array}$ & Medicago sativa (Alfalfa) + Iris lactea (Iris) \\
\hline 3 & $\begin{array}{l}\text { Prunus } \quad \text { armeniaca(Apricot) } \\
\text { Juglansregia(Walnut) }+ \text { Salix alba (Willow) }\end{array}$ & Medicago sativa (Alfalfa) \\
\hline 4 & $\begin{array}{l}\text { Prunus armeniaca(Apricot) }+ \text { Salix alba } \\
\text { (Willow) }\end{array}$ & Medicago falcata(Alfalfa) + Iris lactea(Iris) \\
\hline \multicolumn{3}{|c|}{ Agrihorticulture land use system } \\
\hline Sr. No. & Agriculture crop & Fruit trees \\
\hline 1 & Triticum aestivum(Wheat) & $\begin{array}{l}\text { Prunus armeniaca(Apricot) } \\
\text { domestica(Apple) }\end{array}$ \\
\hline 2 & $\begin{array}{l}\text { Solanum tuberosum(Potato) }+ \text { Pisum } \\
\text { sativum }(\text { Pea })+\text { Phaseolus vulgaris }(\text { Beans })\end{array}$ & $\begin{array}{l}\text { Prunus armeniaca(Apricot)+ } \\
\text { domestica(Apple) }\end{array}$ \\
\hline 3 & $\begin{array}{l}\text { Brassica } \quad \begin{aligned} \text { rapa(Turnip) } \\
\text { lycopersicon(Tomato) }\end{aligned} \\
\text { (Tolanum }\end{array}$ & $\begin{array}{l}\text { Prunus armeniaca(Apricot) }+ \text { Morus alba } \\
\text { (Mulberry) }+ \text { Juglansregia(Walnut) }\end{array}$ \\
\hline
\end{tabular}

Fig.1 Location map of the study area

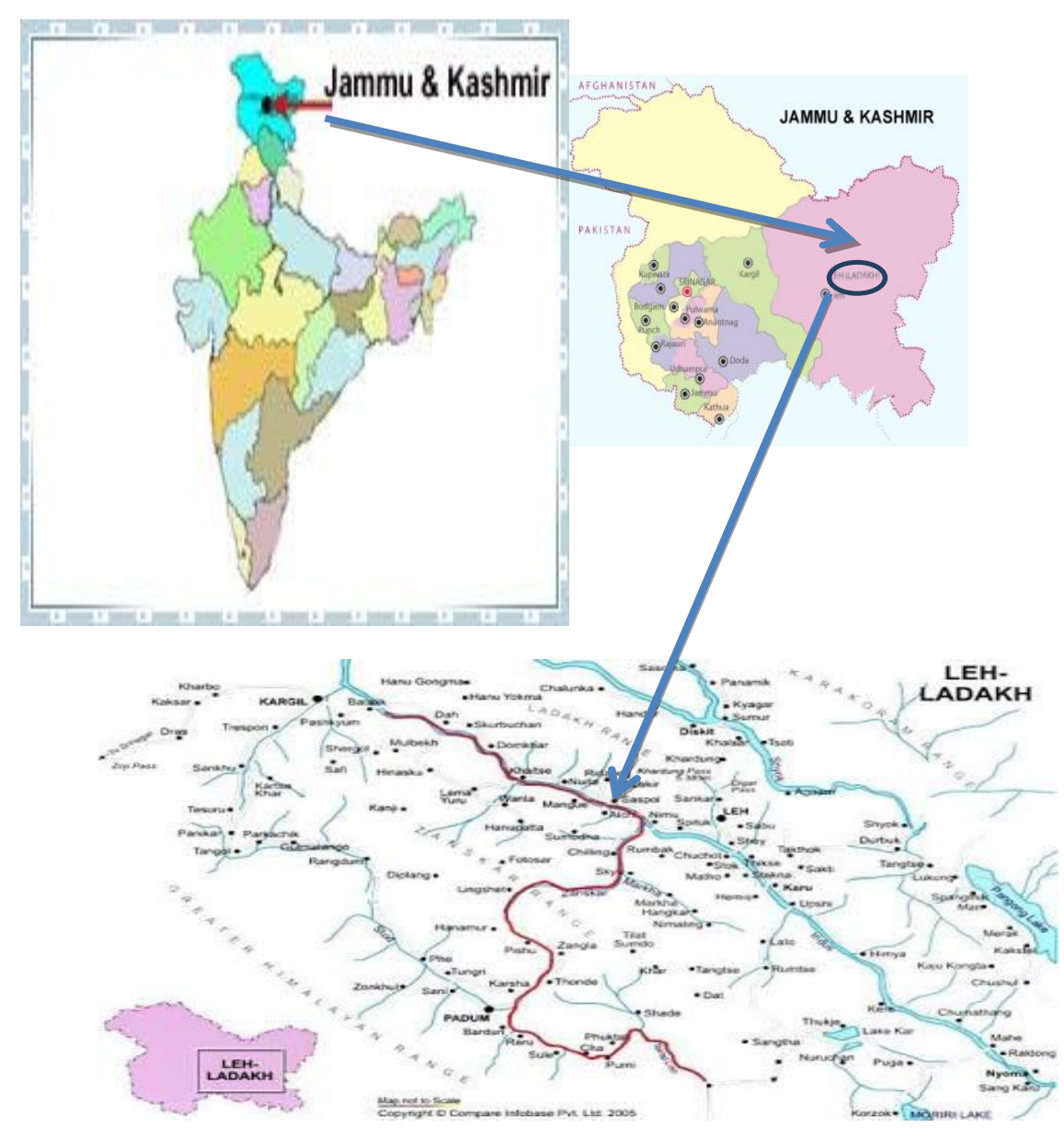

Plate.1 Agriculture land use (wheat) Horticulture land use (apricot trees) 


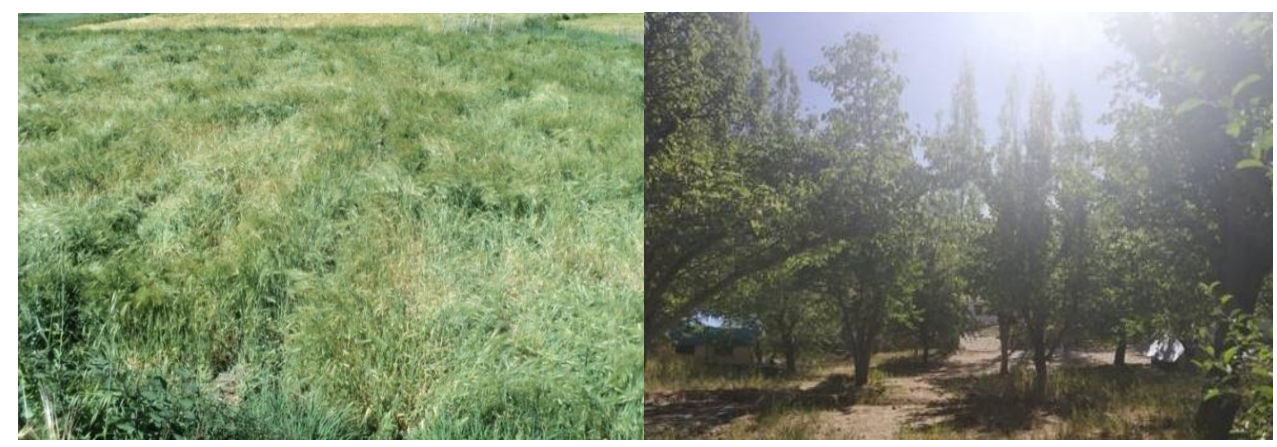

Plate.2 Agrisilviculture land use (wheat+poplar) Hortisilvipasture apricot+salix+alfalfa)
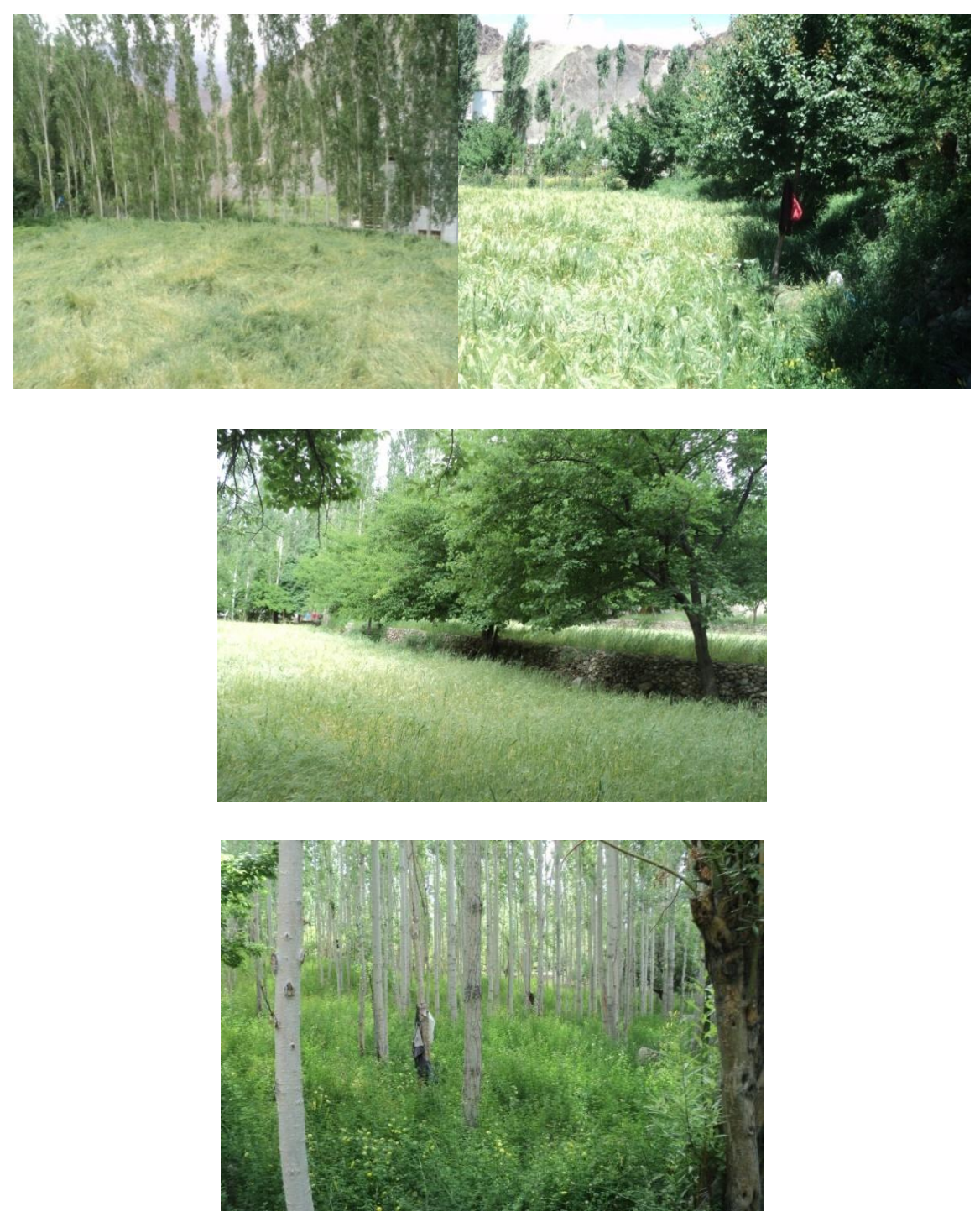
Plate.3 Agrihorticulture (wheat+apricot) Silvipasture (poplar+alfalfa) Hortisilvipasture (apricot+salix+alfalfa)

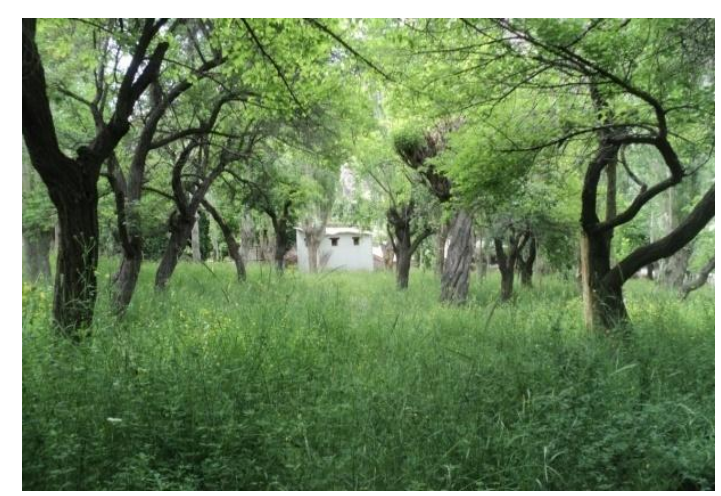

The pasture components consisted of Medicago sativa, Medicago falcata and Iris lactea. The different tree species in hortisilvipasture land use system were Prunus armeniaca, Malus domestica, Juglansregia, Populus nigra and Salix alba, whereas, Medicago sativa, Medicago falcata and Iris lacteal constituted the pasture component. In agrihorticulture land use system, the farmers mainly raised six agriculture crops viz. Wheat, Potato, Pea, Beans, Turnip and Tomato. The tree component comprised of trees of Apricot, Apple and Mulberry (Table5).

In Ladakh region, traditional agroforestry system in the form of agrisilvicultural system existed since time immemorial. Agriculture fields usually had boundary plantations of multipurpose trees of Willow (Salix spp.) and Poplar (Populus spp.). Silvipasture system consisting of Poplar and Salix trees along with Alfalfa was the dominant system. The major agroforestry systems identified by them were: Poplar+ food crops/Potato/vegetables/ fodder crops; Salix + food crops/ Potato/ vegetable/fodder crops; Apricot/Apple/ Walnut + food crops/Potato/vegetable/fodder crops; food crops/Potato/vegetable + fodder crops (Bhat et al., 2015). Nayak et al., (2011) identified and categorized five types of agroforestry systems in cold deserts of Lahaul and Spiti area. These identified systems were agrihorticulture, agrisilviculture, agrisilvipasture, silvipasture and hortipasture. The major tree species of the area were Willow, Poplar and Apple. Goswami (2008) reported five agroforestry systems viz., agrisilvihorticulture (Maize, Wheat, Black gram, Grewia, Ficus and Pear), agrihortisilviculture (Wheat, Rajmash, Tomato, Pomegranate and Grewia), agrisilviculture (Maize, Barley and Ficus and Grewia), agrihorticulture (Maize, Capsicum, Tomato, Plum and Pear) and silvipasture (Pinus, Acacia and grasses) in Kwaalkhad watershed in district Solan, Himachal Pradesh. Rajput (2010) reported four agroforestry systems viz, agrihorticulture having Maize, Black gram, Cauliflower and Apple; agrisilviculture with Maize, Wheat, Grewia and Celtis; agrihorticulture with Maize, Wheat, Tomato, Apple and Grewia and silvipasture havingGrewia, Chir pine, Bauhinia and grasses in Kullu valley of Himachal Pradesh.

The studies on the Identification of different land use systems in Leh region of Himalayan cold desert concluded that the farmers in Leh region practiced different land use systems viz. agriculture, horticulture, agrisilviculture, agrihortisilviculture, hortisilvipasture, silvipasture and agrihorticulture. The component diversity in these systems did not show significant variation in Khaltsi and Saspol block. The cereals, pulses and 
vegetable crops grown by farmers in the area are a result of their inherited experience ably supported by scientific output.

\section{Acknowledgments}

The authors are thankful to the Department of Silvicultre and Agroforestry in Dr Yashwant Singh Parmar University of Horticulture and Forestry NauniSolan HP India for providing the necessary facilities and moral support to conduct the study.

\section{References}

Atul, P. and Khosla P.K., 1994.Himalayan agro-ecosystems status- Case study. Biological Agriculture and Horticulture 104: 271-286.

Bhat, R. K, Raghuvanshi, M.S., and Kalia, R.K. 2015. Achieving sustainable livelihood in cold arid regions of India through multienterprise options. Annals of Arid Zone 54:1-12.

Goswami, S. 2008. Appraisal of agroforestry land use system for their carbon sequestration potential. M Sc Thesis. Department of Silviculture and Agroforestry, Dr YS Parmar University of Horticulture and Forestry, Nauni, Solan. 134p.

Gupta, R.D., and Arora. S. 2016. Ecology, soil and crop management for livelihood in Ladakh region: an overview. Journal of Soil and Water Conservation 2:178185.

Lillesand, T.M., and Kiefer, R.W. 2000. Remote Sensing and Image Interpretation. John Wiley and Sons. Inc., New Jersey. 95p.

Nair, P.K.R., 1985.Classification of agroforestry systems. Agroforestry Systems 3:97-128.

Nayak, P.D., Bawa, R. and Gupta, T. 2011. Agroforestry systems of Lahaul and Spiti district of Himachal Pradesh, western Himalaya. Indian Journal of Ecology 38:129-131.

Rajput, B.S., 2010Bio-economic appraisal and carbon sequestration potential of different land use systems in temperate north-western Himalayas. Ph.D. Thesis. Department of Silviculture and Agroforestry, Dr YS Parmar University of Horticulture and Forestry, Nauni, Solan. 169p.

Tiwari, P.C.,2000 Land use changes in Himalaya and their impact on the plains economy: Need for sustainable land use. Land Use Policy 17:101-111.

Toky, O.P., Kumar P and Khosla PK. (1989) Structure and function of traditional agroforestry systems in the western Himalaya. I. Biomass \& productivity. Agroforestry Systems 9: 47-70.

\section{How to cite this article:}

Jigmet Namgial, Mukesh Prabhakar, RohitBishist, Krishan Lal Gautam, Harish Sharma and Archana Sharma. 2020. Identification of Different Land Use Systems in Leh Region of Himalayan Cold Desert. Int.J.Curr.Microbiol.App.Sci. 9(11): 2768-2778. doi: https://doi.org/10.20546/ijcmas.2020.911.336 